\title{
Lymphoceles following Second-Look Laparotomy for Ovarian Cancer
}

\author{
Keith Y. Terada, M.D., and James A. Roberts, M.D. \\ Department of Obstetrics and Gynecology, University of Michigan School of Medicine, Women's \\ Hospital, 1500 East Medical Center Drive, Ann Arbor, Michigan 48109
}

Received May 28, 1986

\begin{abstract}
In patients treated for ovarian cancer it is crucial to distinguish recurrent malignancy from a benign process. Presented herein is a patient who developed bilateral pelvic lymphoceles following a second-look laparotomy for ovarian cancer. Indications for surgical intervention are discussed and the preferred surgical approach is outlined. (c) 1988 Academic Press, Inc.
\end{abstract}

Retroperitoneal pelvic and periaortic lymph node dissections have a major role in the management of gynecologic malignancies. One well-known complication of the procedure is the formation of retroperitoneal lymphoceles. This has been noted to occur in approximately $5 \%$ of patients who have undergone a pelvic or periaortic lymphadenectomy in conjunction with radiation therapy for cervical cancer [1]. The incidence of this complication following operations for ovarian cancer is unknown. Occasional cases have been reported following primary operations for ovarian cancer [2], but not as a complication of second-look operations.

Lymphoceles may develop following any surgical procedure involving extensive retroperitoneal dissection about the great vessels of the pelvis. A pelvic mass detected in a patient with ovarian carcinoma cannot always be assumed to represent tumor recurrence. Presented herein is a patient who developed bilateral pelvic lymphoceles as an unusual sequela to second-look laparotomy for ovarian carcinoma.

\section{CASE REPORT}

The patient is a 62-year-old female who underwent an exploratory laparotomy, total abdominal hysterectomy, and bilateral salpingo-oophorectomy in 1984 for a stage III moderately differentiated papillary serous cystadenocarcinoma of the ovary. Operative findings revealed a large solid cystic pelvic mass with multiple peritoneal implants and ascites; an optimal resection was carried out. The patient then received 12 courses of combination chemotherapy consisting of cis-platinum, Adriamycin, Cytoxan. Following this, the patient was clinically free of disease 
and underwent a second-look laparotomy. Multiple biopsies including selective pelvic and periarotic lymph node biopsies were all negative for carcinoma. A closed suction drain was left in the retroperitoneal space until the fifth postoperative day; drainage was minimal at the time of removal. The postoperative course was uneventful. Two months later the patient developed bilateral cystic pelvic masses confirmed by CT scan and measuring $9 \times 6 \times 8 \mathrm{~cm}$ on the left and $6.5 \times 4$ $\times 4 \mathrm{~cm}$ on the right. A small amount of intraperitoneal fluid was also noted on CT. Ultrasound-guided needle aspiration of both cysts was carried out; cytologic evaluation of the fluid was negative for malignancy. Subsequently an exploratory laparotomy was done. Bilateral lymphoceles were recognized; there was no evidence of recurrent malignancy. Open drainage and marsupialization to the peritoneal cavity were carried out. The patient was discharged on her fifth day in excellent condition.

\section{DISCUSSION}

The incidence of lymphoceles following operations for ovarian carcinoma is unknown. Various series on second-look laparotomies in patients with ovarian carcinoma have not recorded this as a complication of the procedure [3-5]. In any patient with ovarian carcinoma it is crucial to distinguish a benign process from recurrent disease; a pelvic mass detected by bimanual examination cannot be assumed to represent recurrent tumor. CT scan and ultrasound may be valuable adjuncts in this regard. In this patient, the radiographic appearance of bilateral, discreet, thin-walled, homogeneous cysts, adjacent to the psoas muscle on the left and indenting the bladder on the right, suggested that these were lymphoceles. However, the risk of recurrent carcinoma after a negative second-look operation may be as high as $50 \%$ [5]. Clearly an aggressive approach is indicated, particularly since recurrent tumor may present with characteristics resembling lymphoceles [6].

Lymphoceles presumably arise from surgical transection and continued drainage of the afferent lymphatic channels. Risk factors include more extended lymphadenectomy, tumor metastasis to the nodes, prior irradiation, and heparin thromboembolism prophylaxis [7]. Although retroperitoneal closed suction drainage was utilized in this patient, it is not clear whether this increases [8] or decreases [9] the risk of lymphocele formation. The authors currently utilize closed suction drainage for any case involving extended retroperitoneal dissection. Some surgeons, however, feel that suction drainage promotes continued afferent lymphatic flow and therefore should be avoided.

Most lymphoceles become apparent within 3 weeks of surgery. They may be entirely asymptomatic; probably a large number are subclinical and regress spontaneously. Symptoms associated with clinically significant lymphocysts may be abdominal pain, edema of the genitalia or lower extremity, or pressure symptoms secondary to compression of the bladder, ureter, or sigmoid colon. Pelvic vein thrombosis has been reported to occur as a complication of pelvic lymphocysts [10].

Generally, conservative measures are sufficient to treat the majority of lym- 
phoceles, since spontaneous regression usually occurs. Indications for operative intervention include severe pain, massive leg edema, suspicion of recurrent malignancy, or urinary tract obstruction with renal compromise or secondary pyelonephritis. Repeated percutaneous aspiration is not recommended because of the risk of introducing infection.

It is crucial to recognize a lymphocele at the time of laparotomy, since attempts to resect the mass may lead to excessive bleeding from the great pelvic vessels, which constitute the lateral wall of the "cyst." Open drainage is the preferred surgical procedure [11]. A transperitoneal window is created, thereby effectively marsupializing the lymphocele cavity. An extraperitoneal approach with external marsupialization has been suggested as an alternative approach [12].

In summary, patients with ovarian carcinoma presenting with a pelvic mass cannot be assumed to have recurrent disease. An aggressive approach to evaluation is indicated. At the time of laparotomy it is crucial to recognize a lymphocele as such; open drainage and marsupialization is the preferred surgical approach.

\section{REFERENCES}

1. Morton, D. G., Lagasse, L. D., Moore, J. G., Jacobs, M., and Amromin, G. D. Pelvic lymphadenectomy following radiation in cervical carcinoma, Amer. J. Obstet. Gynecol. 88, 932 (1964).

2. Helmkamp, B. F., Krebs, H. G., Isikoff, M. B., Poliakoff, S. R., and Averette, H. E. Paraaortic lymphocyst, Amer. J. Obstet. Gynecol. 138, 395 (1980).

3. Smith, J. P., Delgado, G., and Rutledge, F. Second-look operation in ovarian carcinoma, Cancer 38,1438 (1976).

4. Berek, J. S., Hacker, N. F., Lagasse, L. D., Poth, T., Resnick, B., and Nieberg, R. K. Second look laparotomy in stage III epithelial ovarian cancer: Clinical variables associated with disease status, Obstet. Gynecol. 64, 207 (1984).

5. Koberts, W. S., Hodel, K., Rich, W. M., and DiSaia, P. J. Second-look laparotomy in the management of gynecologic malignancies, Gynecol. Oncol. 13, 345 (1982).

6. Cantrell, C. J., and Wilkinson, E. J. Recurrent squamous cell carcinoma of the cervix within pelvic-abdominal lymphocyst, Obstet. Gynecol. 62, 530 (1983).

7. Persky, L., Kursh, E. D., and Feldman, S. Extrinsic obstruction of the ureter, in Campbell's urology (J. H. Harrison, R. F. Gittes, A. D. Perlmutter, T. A. Stamey, and P. C. Walsh, Eds.), Saunders, Philadelphia, 4th ed. (1978).

8. Rutledge, F., Dodd, G. D., and Kasilag, F. B. Lymphocysts: A complication of radical pelvic surgery, Amer. J. Obstet. Gynecol. 77, 1165 (1959).

9. Symmonds, R. E., and Pratt, J. H. Prevention of fistulas and lymphocysts in radical hysterectomy, Obstet. Gynecol. 17, 57 (1961).

10. Clarke-Pearson, D. L., Synon, I. S., and Creasman, W. T. Significant venous thromboembolism caused by pelvic lymphocysts: Diagnosis and management, Gynecol. Oncol. 13, 136 (1982).

11. Byron, R. L., Yonemoto, R. H., Davajan, V., Townsend, D., Bashore, R., and Morton, D. G. Lymphocysts: Surgical correction and prevention, Amer. J. Obstet. Gynecol. 94, 203 (1966).

12. Livingston, W. D., Confer, D. J., and Smith, R. B. Large lymphoceles resulting from retroperitoneal lymphadenectomy, J. Urol. 124, 543 (1980). 